\title{
Diferenciación del vino a través del terroir: el caso de la DOP Campo de Borja (España)
}

\author{
Miguel LorenteBlasco ${ }^{1}$ y Ernesto Franco Aladrén ${ }^{2}$ \\ ${ }^{1}$ Grupo de Estudios de Desarrollo Territorial, Universidad de Zaragoza (GEDETUZ). España. \\ mlorenteb@yhoo.es \\ ${ }^{2}$ Centro de Transferencia Agroalimentaria - Gobierno Aragón. Unidad de Enología. Avda. de Movera, \\ s/n, 50194, Zaragoza (España).efranco@aragon.es
}

\begin{abstract}
The Regulatory Council of the DOP Campo de Borja (Spain) promoted a zoning study aimed at knowing the geographical environment and its effect on the quality of wines, in order to delimit the space in Units of Terroir (UT) base to apply a model Productive and commercial reasoned following the concept terroir. It was to seek the specialization and recognition of wine values linked to the territory of origin. The study of the medium, reflected in a map of cartographic soil units (SMU) to scale 1:25.000, was accompanied by an oenological study carried out during three vintages to know the variability of the oenological capacities of the SMU. The results indicate that in the DOP have obtained different types of units of terroir depending on the characteristics of the mistelas used to classify and delimit territorially Units of Terroir.
\end{abstract}

\section{Introducción}

El término francés terroir, utilizado en todo el mundo, expresa el fenómeno geográfico que rige la Denominación de Origen (DO), arraigado en la idea de hacer inseparables la producción y el territorio de origen del vino. Del mismo se deriva la servidumbre de entender el vino como el resultado de una cultura emanada de las interacciones producidas en la Naturaleza entre el medio y la vid, y cuyo resultado se manifiesta en las características distintivas de los vinos. Se deduce, por tanto, que la aplicación coherente de la DO va unida a la idea del terroir, lo que lleva implícito conocer el medio y su potencial enológico a través de la zonificación, y utilizar este fenómeno en la producción y comercialización del vino.

En Europa la producción de vinos de calidad se centra en un sistema de distribución territorial basado en DOP e IGP. A este respecto Huglin (1978) consideraba que las DO se deben basar en la delimitación de zonas con condiciones ecológicas particularmente privilegiadas 0 simplemente originales.

Se considera zonificación, en un sentido amplio, a la división racional de un área geográfica en zonas homogéneas de acuerdo a ciertos criterios. Desde el punto de vista de la producción enológica la zonificación se concibe como la investigación del territorio para repartirlo en zonas relativamente homogéneas, como consecuencia de la interacción entre el viñedo y el medio ambiente (Fregoni et al., 1998). Por tanto, la aplicación coherente y razonada de la normativa sobre la DO implica la zonificación territorial, con el fin de determinar los terrenos de especial aptitud para el cultivo de la vid que deben constituir los límites de todos los niveles de las DOP.
Un estudio realizado en la DOP Campo de Borja con el objetivo de promover su desarrollo, indicaba que para mejorar su posicionamiento comercial se debía avanzar en especialización y en el reconocimiento de valores del vino vinculados a el territorio de origen. Esto llevó al Consejo Regulador a promover un trabajo de zonificación destinado a conocer el medio geográfico y su efecto en la calidad de los vinos con el fin de delimitar el espacio en Unidades de Terroir (UT), base para aplicar un modelo productivo y comercial razonado siguiendo el concepto terroir.

El trabajo pretendía proporcionar recursos técnicos a los agentes de la producción, al mismo tiempo que aportaba justificación ideológica (la DO y el terroir son una ideología en el modo de concebir el vino), pero se sabía de las limitaciones que surgen a la hora de aplicar las innovaciones en las zonas productoras, especialmente en el medio rural. Como el trabajo de la DOP Campo de Borja pretendía ser una herramienta para promover el desarrollo, y es sabido que no basta con que lo nuevo sea mejor que lo existente sino que debe ser integrable en la cultura de los que la van a adoptar, en paralelo al trabajo de zonificación se desarrollaron una serie de actividades de comunicación encaminadas a producir cambios en el statu quo, difundiendo los conocimientos mediante conferencias, salidas al campo y cursos para los técnicos sobre manejos de SIG, etc.

\section{Material y métodos}

El estudio se estructura tomando la vid como elemento vivo que se desarrolla afectado por el medio geográfico (con sus factores naturales y humanos), y que, como consecuencia de esta interacción, produce la materia prima del vino con características intrínsecas particulares. Este proceso biológico nos lleva a analizar el 
medio geográfico para delimitar las zonas homogéneas con incidencia en el desarrollo de la vid, y las características enológicas de estas unidades de suelo para determinar las Unidades de Terroir (UT) con posibilidad de ser utilizadas de un modo razonado en la producción de vino. Por tanto, se asocia el término de zonificación a subdivisión (clasificación) de un territorio en base a las características ecopedológicas y topográficas (Fregoni et $a l, 1998), \mathrm{y}$ al resultado enológico fruto de la interacción vid-medio.

Finalmente, esta información se une al SIGPAC y al Registro Vitícola que recoge los datos relacionados con las parcelas cultivadas de vid, permitiendo abordar el análisis estadístico desde la perspectiva del medio geográfico, de la caracterización enológica y del viñedo, para establecer las UT. La interrelación de las tablas de atributos de las capas permite tener información, a escala parcelaria, de todos los factores que afectan al desarrollo del viñedo y a las características enológicas, permitiendo una gestión razonada de la producción de acuerdo a los criterios del terroir.

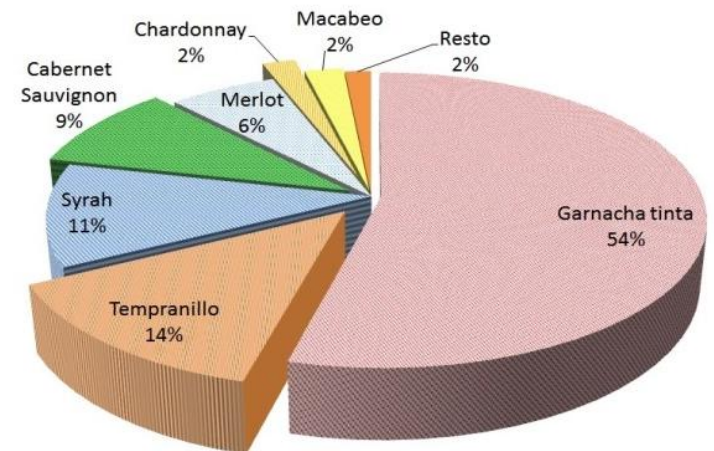

Figura 1. Ocupación de las variedades en la DOP Campo de Borja.

El territorio de la DOP tiene una superficie de 65.300 hectáreas y el viñedo ocupa 6.661 hectáreas repartidas en 10.074 parcelas, lo que representa una dimensión parcelaria media de las viñas de 0,6 ha y un índice de ocupación del viñedo en la zona de 9,5\%. La variedad garnacha es la tradicional de la DOP y ocupa el $55 \%$ de la superficie, seguida a gran distancia de otras variedades de reciente implantación (Figura 1). La preponderancia e interés de la garnacha hace que el estudio enológico se realice sobre esta variedad.

\subsection{Estudio del medio}

El método utilizado en el estudio del medio se ha aplicado en distintas DOP de España y otros países por Sotés y Gómez-Miguel (1993-2015). Se trata de un análisis geográfico del territorio en el que el resultado final es un mapa cuyas unidades cartográficas (SMU) sintetizan las relaciones entre clima, litología, geoformas y serie de suelos. El manejo de la información generada en las capas tratadas por un Sistema de Información Geográfica (SIG) da como resultado la cuantificación de los contenidos y la posibilidad de su tratamiento estadístico.

En el estudio del medio se tuvo en cuenta el clima, la topografía, la geología y los suelos, según Soil Survey
Manual y Soil Taxonomy USDA (1990-2014). Como base de reconocimiento se utilizaron fotogramas aéreos a escala 1:20.000, ortofotos, mapa topográfico nacional, mapas geológicos del IGME y mapa forestal español. En el trabajo de campo se describieron 519 perfiles y 1.560

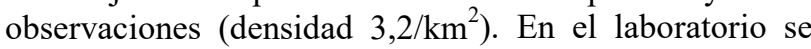
analizaron 2.309 muestras.

El mapa final a escala 1:25.000 está constituido por SMU cuyos contenidos reflejan los tipos de suelos en unidades taxonómicas (STU) o taxa (Figura 2).

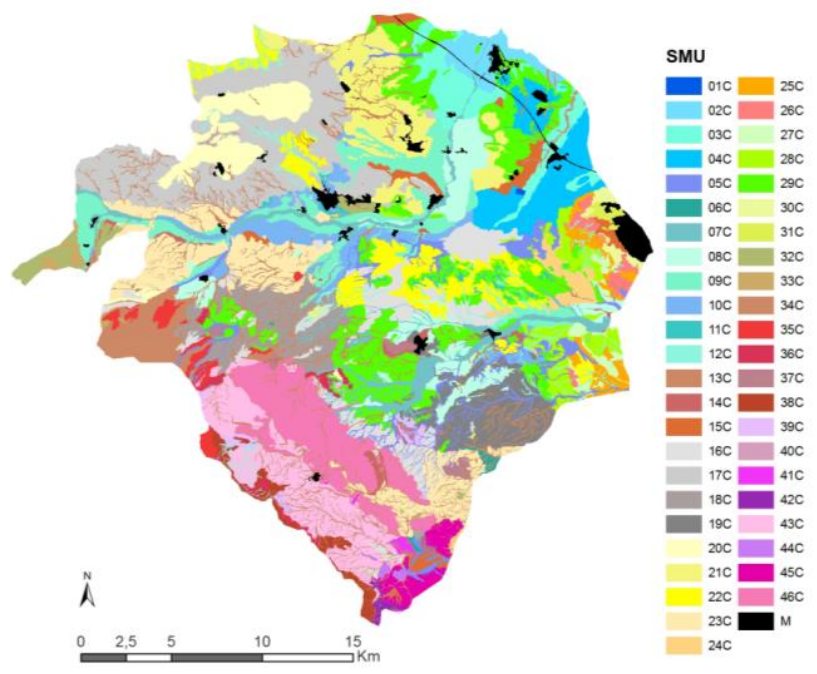

Figura 2. Mapa de suelos en SMU. Fuentes: Gomez-Miguel et al, 2015)

\subsection{Los suelos de la DOP Campo de Borja}

El mapa de suelo de la DOP está compuesto de 46 SMU aunque diez de ellas ocupan prácticamente el $50 \%$ de la superficie. El $64 \%$ del viñedo se encuentra en el 33 $\%$ de la superficie compuesta por ocho SMU. Tomando las unidades taxonómicas principales (STU) y secundarias de suelo, como representantes de mayor ocupación de las SMU, los resultados muestran que dos STU destacan sobre las demás, puesto que soportan un tercio del viñedo. Las seis STU siguientes en ocupación albergan al $45 \%$ y otras siete el $17 \%$ (Figura 3). Esto nos demuestra que los viticultores han seleccionado las tierras de forma empírica a la hora de plantar las viñas, Lorente (2017).

\subsection{Estudio enológico}

Definidas las SMU el siguiente paso fue verificar su potencial enológico para descubrir el vínculo entre el vino y el lugar donde se producen las uvas. Pero abordar un estudio de estas características supone manejar un número elevado de parcelas durante varios años y disponer de medios técnicos adecuados. En nuestro caso solo disponíamos de recipientes de poco volumen donde las fermentaciones resulta difícil controlar e introducen factores que no están directamente relacionados con el medio geográfico y pueden desvirtuar el vínculo entre el vino y el territorio, por lo que los resultados a veces resultan erróneos. Por ello se opta por una vinificación sin fermentación alcohólica, mistela, que se lleva acabo en depósitos de 25 litros, tal como se describe en el trabajo de Franco y Lorente (2018). 
Para el estudio se tomaron 60 parcelas de la variedad garnacha tinta con el portainjerto $\mathrm{R}-110$, en secano $\mathrm{y}$ plantadas antes del año 2000, de cuyas uvas se elaboraron 60 mistelas en depósitos de 25 litros y 12 vinos en depósitos de 1.000 litros de parcelas que también se obtenían mistelas. El estudio se realizó en los años 2012, 2013 y 2014. Este método se ajusta a los fines perseguidos puesto que se buscaba determinar la expresión de aromas primarios de la uva, y para ello no era necesario realizar la fermentación alcohólica.

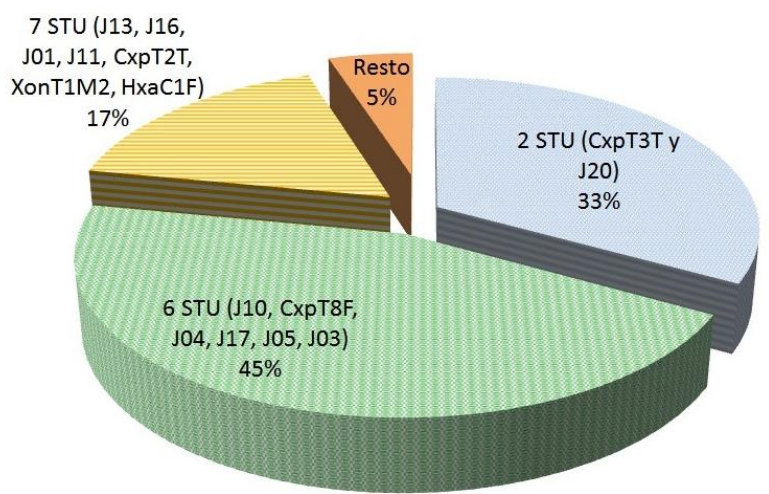

Figura 3. Ocupación del viñedo por STU

Las mistelas y vinos fueron sometidos a análisis físico-químico y sensoriales, y los resultados de todas parcelas fueron tratados estadísticamente para clasificarlos y ver si se formaban grupos diferentes con sus características. Esta clasificación unida a la información del mapa de suelos permitiría conocer la tendencia enológica de cada SMU y determinar el vínculo entre el vino y el territorio.

Hay que destacar que a lo largo de los tres años de ensayos se produjeron una serie de incidencias por diversos motivos que impidieron realizar el trabajo de acuerdo a los protocolos previstos. En 2013 se produjo un fuerte corrimiento de la flor que mermó la cosecha en numerosas parcelas, hasta el punto de impedir realizar algunas vinificaciones. En consecuencia, 24 parcelas de mistela disponen de tres resultados, 27 dos resultados y 17 solamente de un año.

\subsection{Estudio estadístico}

Con los resultados analíticos obtenidos se buscaba comprobar, mediante análisis factorial, si las diferencias entre los valores de las parcelas eran significativas, ya que en caso contrario se produciría un caso de uniformidad. Igualmente se quería conocer si los valores de las parcelas podían ser clasificados en grupos homogéneos para ver posteriormente si existía correlación con las SMU. Se pretendía establecer un modelo que, a partir de las variables analizadas en las mistelas, permitiera definir sus características, comprobar su variabilidad espacial y relacionarlas con las unidades cartográficas de suelo. Para ello se realizó un tratamiento estadístico utilizando la técnica de análisis multivariante (Júdez 1989, Dolédec 1995), en el que se consideraban variables los valores de los elementos organolépticos y parámetros del análisis físico-químico obtenidos mediante la metodología descrita Franco y Lorente (2018).

En el estudio estadístico se utilizaron técnicas de análisis de regresión, análisis exploratorio de Tukey (estadística descriptiva) y análisis multivariante (análisis de componentes principales -ACP-, clasificación automática según diferentes criterios de distancia). Dichas técnicas se aplicaron utilizando el programa informático SPSS.

\subsection{Relación entre las características enológicas de las mistelas y las SMU}

Tras determinar a través de la clasificación automática los grupos en los que las mistelas se clasifican según sus características enológicas y la pertenencia de cada parcela a un grupo concreto, con la herramienta de geoprocesamiento de ArcGis se cruzan las capas en formato vectorial que recogen las unidades cartográficas de suelo (SMU) y las parcelas de ensayo extraídas de los recintos del SIGPAC. Esta operación de intersección permite añadir a las parcelas un nuevo campo con la SMU en la que se encontraban.

Las parcelas de cada SMU se agrupan y se puede observar su tendencia enológica a partir de los grupos clasificatorios. Esta operación permite comprobar si en cada SMU las parcelas se clasificaban mayoritariamente en uno o dos grupos concretos o si, por el contrario, esta dominancia no se produce, por lo que en el primer caso se podría entender que existe relación entre el SMU y el tipo de uvas que producen, mientras que en el segundo caso esto aún no quedaba demostrado. Se considera grupo dominante cuando más del $50 \%$ de las observaciones se clasifican en ese grupo. En algunos casos esa dominancia la producen dos grupos contiguos con algunas características comunes y en otros, normalmente por falta de observaciones, no se aprecia ninguna dominancia.

\section{Resultados}

\subsection{Analíticos y sensoriales de las mistelas}

El análisis de componentes principales (ACP) de la concentración de azúcar, color e IPT, muestra un efecto de la añada muy moderado, a pesa que en las medias se observe diferencia importante que se atribuían a la diferencia de pluviometría, (Franco y Lorente, 2018). En la F-Q como en el sensorial se ve que las añadas de 2013 y 2014 son más parecidas entre sí, coincidiendo con una pluviometría superior a la de 2012. También se aprecia que algunas variables presentan pocas diferencias entre los años, mientras que en otras la variabilidad es mayor Los resultados del ACP de todas las mistelas, con independencia del años y con las variables físicoquímicas más explicativas, intensidad de color e índice de polifenoles totales en la componente 1 , y azúcares en la componente 2, según el trabajo previo de Franco y Lorente (2018), se muestra en la Figura 4, observando en la zona positiva de la componente 2 mayor presencia de las mistelas de 2012, mientras que las de los años 2013 y 2014 están mayoritariamente distribuidas en la zona negativa de la componente 2, la menor lluvia de 2012 
justificaría este comportamiento, sin embargo no podemos asignar diferencia significativas en la analítica de las mistelas en función del año.

El ACP del análisis sensorial indica que la componente 1 engloba los aromas a fruta negra, fruta pasa y tinta, y la componente 2 la fruta roja y el balsámico, según el trabajo previo de Franco y Lorente (2018), en este caso la distribución de las mistelas es más uniforme que con parámetros químicos y tampoco podemos asignar diferencia significativas en el análisis sensorial de las mistelas en función del año, Figura 5.
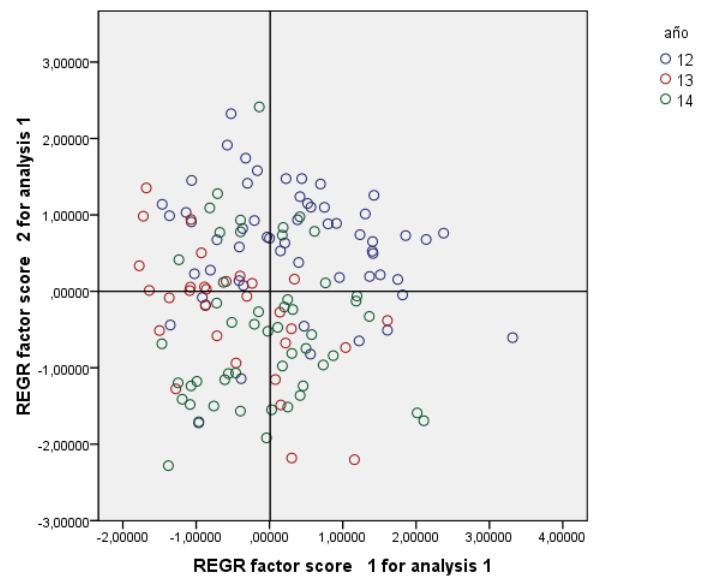

Figura 4. Distribución de las mistelas en ACP de los parámetros analíticos.
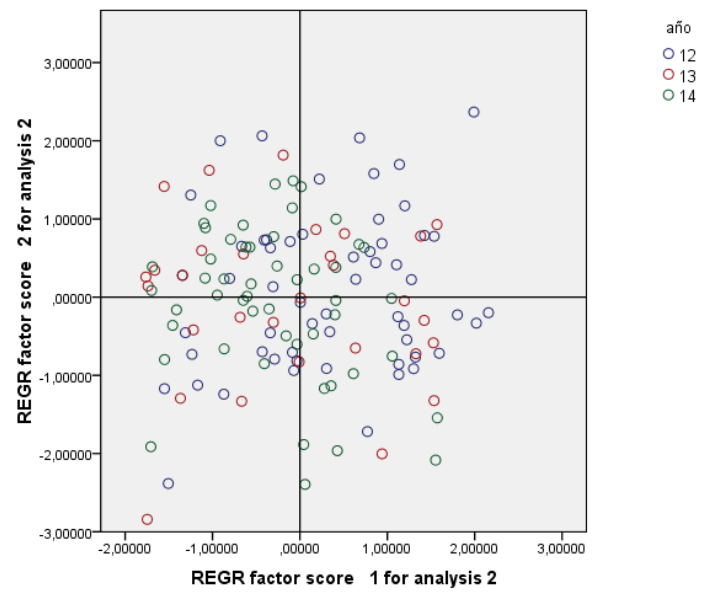

Figura 5. Distribución de las mistelas en ACP de los parámetros sensoriales.

\subsection{Clasificación de las mistelas}

Si bien estadísticamente no podemos hablar de un efecto añada nítido, sí que se observa una influencia de la añada, que condiciona la clasificación de las mistela.

La aplicación de la clasificación automática con la base de los parámetros físico-químicos y del análisis sensorial clasifican las mistelas en cuatro grupos ajustados a los cuadrantes formados por los dos ejes que representan los componentes principales. Ahora bien, la clasificación por cuadrantes es similar en los tres años y solo se representa la clasificación de las mistelas el año 2014.
La distribución de las mistelas en función de los tres parámetros químicos, se muestra en la figura 6 , indica que las mistelas del primer cuadrante se caracterizan por moderado IPT y color, las del segundo cuadrante presentan los máximos valores de IPT y color, las del tercer cuadrante IPT y color son elevadas, inferiores a las del segundo y superiores a primero y cuarto y las del cuarto son las menores en los tres parámetros. Figura 7, remarcar que la concentración de azúcar es muy similar en los cuatro grupos y en consecuencia quien realmente clasifica las mistelas es el IPT y la intensidad colorante.

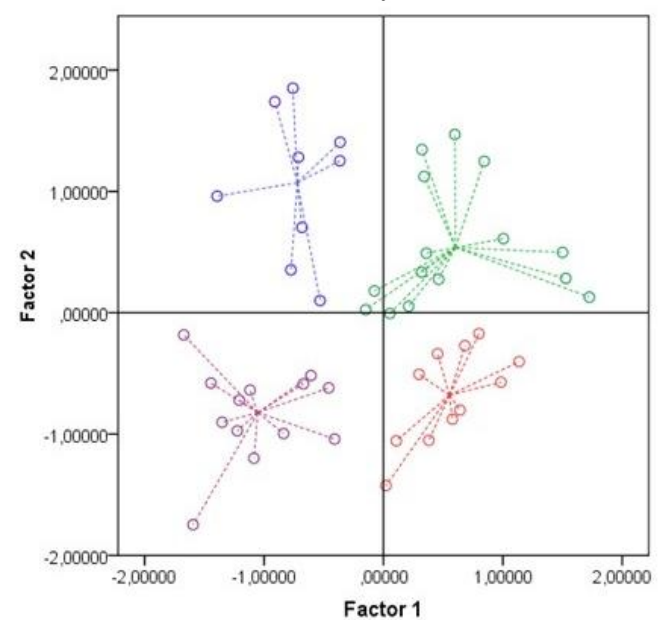

Grupos

1

(1)

3

( 4

Figura 6. Clasificación en grupos de las mistelas en función de los parámetros químicos del año 2014

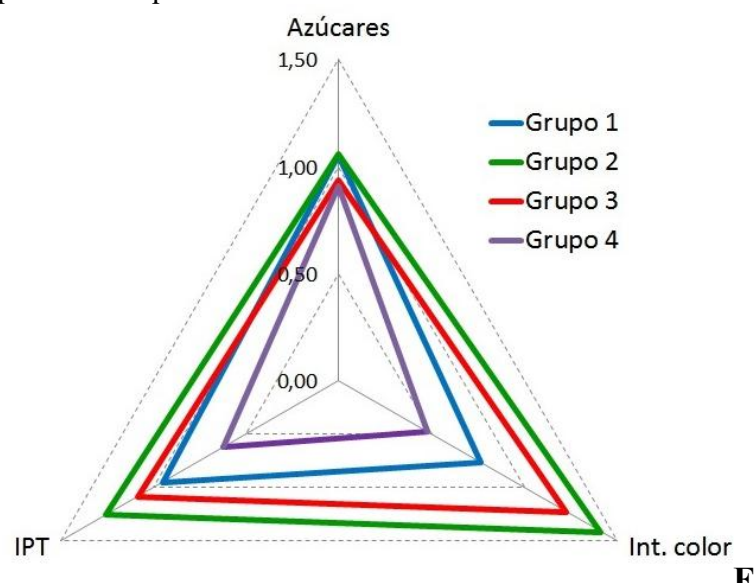

igura 7. Representación de los valores medios de los parámetros químicos de los grupos resultantes de la clasificación. Años 2012, 2013 y 2014.

En el caso del análisis sensorial el grupo uno, del primer cuadrante, se caracteriza por predominar los aromas a fruta roja y balsámico pero con menores valores de la fruta negra y tinta, el grupo dos presenta valores altos en todos los descriptores, el grupo tres los valores bajos son de fruta roja y balsámico, y el grupo cuatro todos los descriptores son bajos, figura 8 .

En la figura 9, se representan los valores de los diferentes descriptores del aroma de las mistelas en función de la clasificación en cuatro grupos, el grupo cuatro es el de valores más bajos de los descriptores y en consecuencia el de menor calidad, mientras el grupo dos presenta los valores más elevados en todos los descriptores menos en la tinta, que el grupo tres es superior. Las diferencias importantes observadas entre los 
valores de los cuatro grupos indican que esta metodología es adecuada para clasificar las mistelas en grupos en función de sus características aromáticas.

Una vez clasificadas las mistelas en cuatro grupos para cada uno de los tres años de estudio, la pregunta es ¿Cuántas mistelas de la misma parcela repiten en el mismo grupo? Con tres observaciones, es decir con tres mistelas de la misma parcela, la probabilidad de que se repita grupo dos o tres años es del $78 \%$ en el sensorial y del $56 \%$ en el químico.

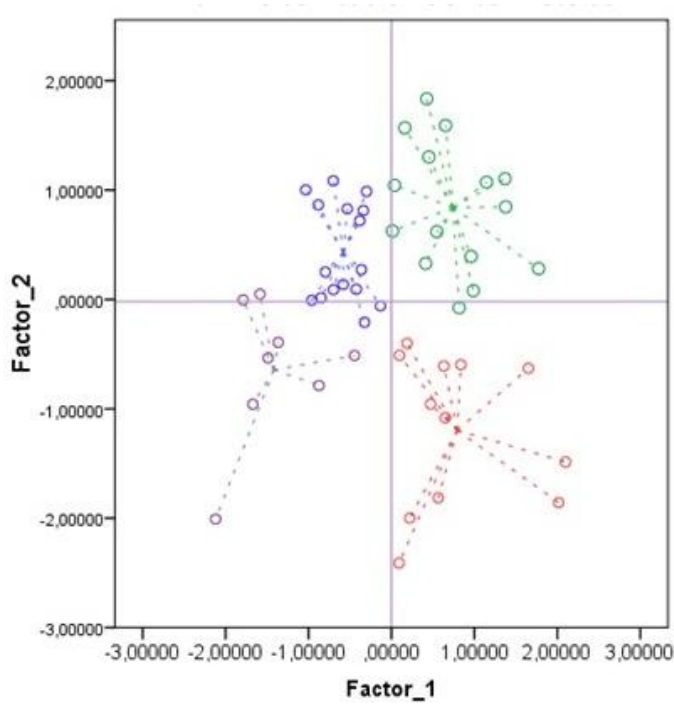

Grupos

1

( 2

○ 3

○ 4

Figura 8. Clasificación en grupos de las mistelas en función de los descriptores sensoriales del año 2014

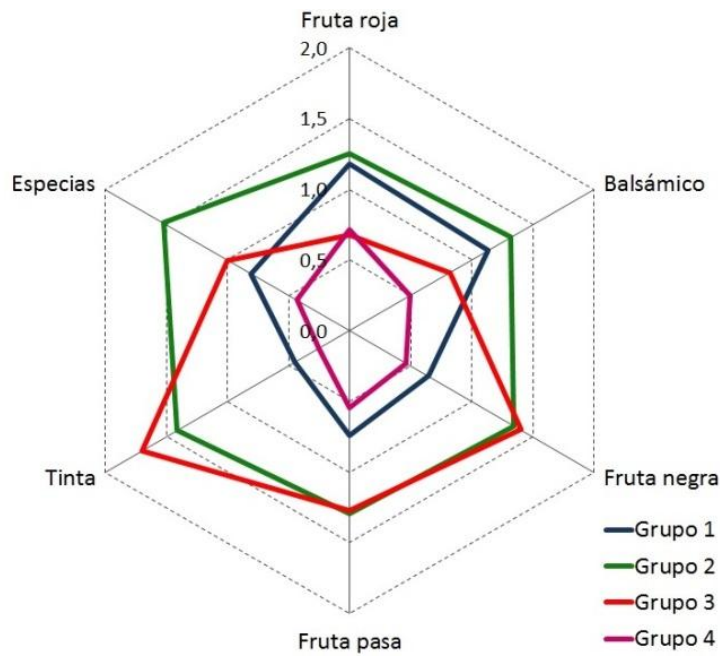

Figura 9. Representación de los valores medios de los descriptores del aroma de los grupos resultantes de la clasificación. Años 2012, 2013 y 2014.

\subsection{Asignación de las parcelas a las SMU, en función de las características enológicas de las mistelas obtenidas.}

Cuando las SMU se asocian a uno o varios grupos enológicos concretos, se puede decir que constituyen una unidad de terroir. Para este estudio se tomaron todas
SMU con parcelas de ensayo excepto las que disponían menos de cuatro observaciones.

Los grupos 1 y 4 mayoritariamente se producen en unidades cartográficas del Cuaternario (fondos de valle y terrazas), mientras que las del Terciario y Triásico se asocian a los grupos 2 y 3 . Esto hace que se produzca una gran similitud entre el mapa de características enológicas y el de Litología.

En la mayoría de los casos las unidades cartográficas tienen más probabilidades de clasificarse en uno o dos grupos concretos, lo que se podría considerar como potencial enológico de la SMU que sirve para definir las UT. Las observaciones de las parcelas cuyos valores del análisis estadístico (ACP) se representan próximas a los ejes, pequeñas variaciones por la añada $u$ otros factores pueden influir en la clasificación, cambiando de grupo, de un año a otro, por tanto, en algunos casos el potencial de las SMU debe considerarse con cierta flexibilidad o considerar que la probabilidad para clasificarse en grupos diferentes es mayor.

Por ello, si en el mapa de SMU se agrupan las unidades que se relacionan con grupos enológicos concretos, se convierte en el mapa de unidades de terroir (UT), así postulamos seis unidades de terroir (UT) que se muestran en la figura 10 , tres de estas unidades se corresponden con los grupos 2,3 y 4, mientras las otras dos comparten grupos, 1-4, y 2-3. En las SMU con menos de 4 observaciones es difícil determinar su potencial enológico por falta de información, son las indicaciones de pocas referencias.

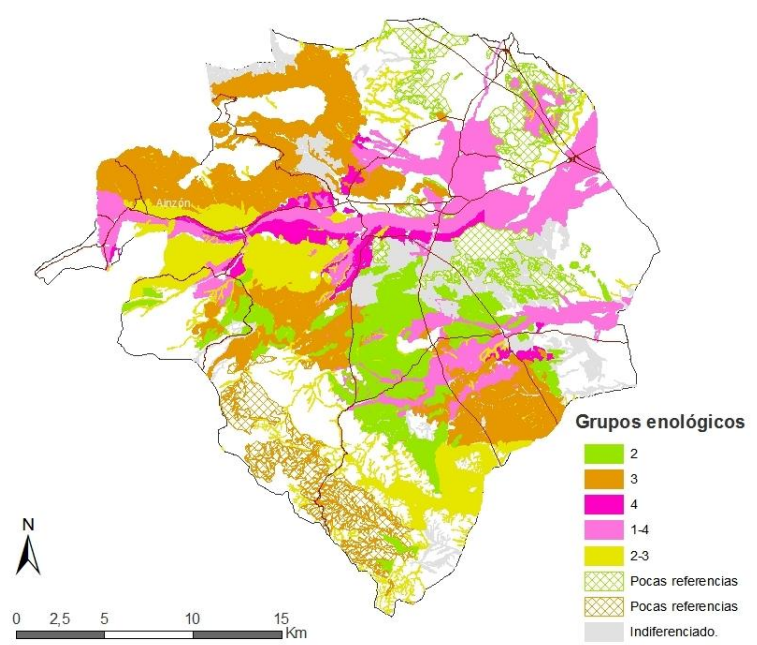

Figura 10. Mapa de Unidades de Terroir, según los grupos enológicos, SMU con al menos cuatro observaciones.

Se ha observado que los vinos de mayor expresión y valor se obtienen en UT cuyas mistelas se han clasificado en el grupo 2 y 3 . A su vez se constata que las zonas clasificadas 1 y 4 , se tiende a arrancar la garnacha por falta de color y calidad organoléptica.

\section{Conclusiones}

$1^{\text {a }}$ La metodología desarrollada para evaluar la potencialidad enológica de los viñedos resulta válida y es sencilla de aplicar, vinificación de mistelas. Esta facilita 
el estudio de las aptitudes de los viñedos a una escala de mayor precisión. La línea de trabajo emprendida ha abierto un camino para continuar profundizando en el estudio del terroir.

$2^{\mathrm{a}}$ En este estudio concreto en la D.O.P. Campo de Borja y durante los años 2012, 2013 y 2014 y con la información obtenida, hemos clasificado las mistelas en cuatro grupos, que se corresponden con características enológicas bien diferenciadas entre los grupos.

$3^{\text {a }}$ Se han postulado seis unidades de terroir (UT), tres se corresponden con los grupos 2,3 y4, mientras las otras dos comparten grupos, 1-4, y 2-3. Con estos datos se observa claramente el efecto terroir en la composición de las mistelas y por tanto en las uvas y en los vinos. Aunque el reducido número de observaciones de algunas SMU impide establecer un modelo predictivo estadísticamente concluyente en estas unidades, por lo que es necesario continuar con el estudio enológico durante un periodo más largo, con el fin de disponer de más información.

$4^{\mathrm{a}}$ No obstante y a pesar de la observación anterior, como conclusión final se puede afirmar que algunas SMU han mostrado su potencial enológico de un modo estable, bien asociándose a un grupo concreto o a dos grupos vecinos con características comunes, se pueden vincular a un tipo de uvas $y$, en consecuencia, a una unidad de terroir.

$5^{\text {a }}$ La variabilidad en la composición de las uvas de las parcelas estudiadas, clasificadas en cuatro grupos según sus características enológicas y sensoriales, nos indica que el territorio de la DOP Campo de Borja, a pesar de su aparente uniformidad, presenta claras diferencias enológicas según las características del medio, posibilitando la implantación de un sistema productivo basado en concepto terroir.

\section{Referencias bibliográficas}

DOLÉDEC, A.-F. (1995). Recherche des composantes principales des terroirs viticoles afin d'elaborer un outil d'aide à la gestión au moyen d'observatoires et de traitements statistiques de données spatialisées. Application au vignoble champenois. Tesis INA P-G.

FRANCO, E， LORENTE,M. (2018). Making mistelas: New methodology for evaluating the oenological characterisctics of Terroir Units. XII Congreso Terroir. Zaragoza.

FREGONI, M. et al (1998). La zonazione viticola della Collina cesenate. Vignevini, 1/2: 39-57

HUGLIN, P. (1978). Nouveau mode d'évaluation des possibilités héliothermiques d'un milieu viticole. Comptes Rendues de l'Académie d'Agriculture. 11171126.

JÚDEZ, A. (1989). Técnicas de análisis de datos multidimensionales. MAPA. Madrid. 301pp.
LORENTE,M. (2017). Zonificación de la D.O.P. Campo de Borja con criterios de terroir. Tesis Doctoral. Universidad de Zaragoza.

SOTÉS,V., GÓMEZ-MIGUEL,V. (1992). Criterios de diferenciación y delimitación de comarcas y regiones vitícolas. Exámen de los factores naturales y humanos que concurren. Vitivinicultura, 3,5: 29-40. 